\title{
Control System Design Technique and Validation for A High- Resolution Mapping Satellite
}

\author{
Chen Chao ${ }^{1,2^{*}}$ Wang ShuYi ${ }^{2}$ LiuJie ${ }^{2}$ Wu SenTang ${ }^{1}$ \\ 1.School of Automation Science and Electrical Engineering, Beihang University, Beijing 100191, China \\ 2. Beijing Institute of Control Engineering, 100190, China
}

\begin{abstract}
With the rapid development of the national economy, the digital city and smart city have become the development trends of China's urbanization, proposing higher and higher requirements for earth mapping. The emergence of high-resolution mapping satellites has satisfied the demand for national economic development. Since China's first satellite measuring scale of 1:10,000 was launched in 2019, thus GF-7 satellite has realized the arc-second attitude measurement accuracy, where the degree of stability reached 10$5 \% / \mathrm{s}$. Based on the author's multi-year research on the design of high-resolution satellite control system, the key technologies and design methods for the high-precision attitude measurement and control system were expounded, the validation results were explained, and the future direction for improving the control precision of the high-resolution mapping satellite was proposed in the end.
\end{abstract}

\section{Introduction}

A visible-light satellite requires high positional accuracy for the plane and elevation of the ground target, which is directly influenced by the satellite attitude determination and orbit determination accuracies, besides the resolution of camera payload. Therefore, the satellite attitude control system becomes crucial. In comparison with the earlystage satellites ${ }^{[1]-[4]}$, the $1: 10,000$ high-resolution mapping satellite has proposed higher requirements for the attitude measurement accuracy of the control subsystem, attitude control precision and stability, satellite maneuverability, service life, reliability, and ground-based validation.

Through an investigation on Chinese and overseas high-precision mapping satellite control system designs $[1],[4]-[6]$, the configuration of the satellite control system varies, to a great extent, with the mapping system ${ }^{[5]}$. The remote sensing satellites equipped with the variable orbit mapping system, including early-stage Quick Bird, and WorldView-4 launched in recent years, are equipped with CMG group, which realizes variable orbit mapping through fast maneuver, or satisfies the stereo mapping demand via fast maneuver, staring imaging, etc. The coorbit mapping is represented by the Japanese ALOS-1 satellite, which is configured with a momentum wheel to realize the co-orbit stereo mapping.

Based on a general survey on mapping satellites in countries outside China, their control systems are all equipped with high-precision star sensors for the attitude measurement, thus realizing the high-precision attitude measurement ${ }^{[8]}$, where American mapping satellites have reached the highest level thanks to their sub-arc second star sensors.

The mapping satellites have a late start in China, and the current civilian mapping satellites are represented by ZY-3 satellites 01,02 and 03 , the stability of the control system reaches $5 \times 10^{-40} / \mathrm{s}$, and the inertial attitude measurement accuracy reaches $0.005^{\circ}{ }^{[7]}$.

Comparing the mapping satellites in both China and foreign countries, the control indexes of the GF-7 satellite launched in 2019 are at advanced levels within the field of mapping satellite. The index comparison results are seen in Table 1.

Table 1 The performance specification comparisons between home and abroad

\begin{tabular}{ll}
\hline Satllite & The performance specification of AOCS \\
\hline GeoEye-1 & Precision: 0.4 arc-second \\
& Stability: $10^{-60} / \mathrm{s}$ \\
Worldview-1 3 & Precision:Less than 0.5 arc-second \\
SPOT-6、7 & Precision: 6.0 arc-second \\
& precision $: 1.44$ arc-second \\
ALOS-1、3 & $\begin{array}{l}\text { Stability: } 5 \times 10^{-4 \circ} / \mathrm{s} \\
\text { precision: } 5 \text { arc-second } \\
5 \times 10^{-4 \circ} / \mathrm{s} \\
\text { ZY-3 }\end{array}$ \\
precision: $0.001^{\circ}$ \\
Stability $: 2 \times 10^{-4 \circ} / \mathrm{s}$
\end{tabular}

AOCS: means Attitude and Orbit Control Syetem 
Aquirements, the GF-7 satellite control subsystem has a system architecture consisting of an extra-high-precision star sensor, which has the function of the star map fusion, a highly steady solar array drive mechanism, and an optimized high-precision and short-delay control platform. Moreover, a satellite-ground large-closed-loop attitude compensation algorithm is designed, and the sensor inorbit parameter calibration technique is adopted to realize the steady in-orbit high-precision operation of the satellite. Based on the design features of comprehensive system supporting facilities and classical functions, the control subsystem implements a code firmware library, which is characterized by the control algorithm standardization, programming modularization, and procedural universalization, under the 3D platform system for the first time, and the functional module code is directly applicable to other satellites; the 8-year service life of the control subsystem is realized through the hardware redundancy and system reconfiguration scheme, and the service life of critical standalone device is verified.

According to the features of the high-resolution mapping satellite, and the difficulties existing in the control system design, the key design methods of the control subsystem, including system architecture, technical solution and reliability design, etc., were summarized, with the emphasis laid on the discussion about the high-precision attitude measurement technique and high-stability attitude control technique for the satellite control subsystem. Meanwhile, the key measurement components and actuators were explained. Based on the satellite's in-orbit operation data, the in-orbit validation results of the performance indexes for the control subsystem and its critical newly developed standalone devices were given.

\section{Control System Design}

\subsection{Task analysis}

Compared with the ordinary remote sensing satellites, the mapping satellites have different index requirements for the control system. The ordinary remote sensing satellites propose high precision and high stability requirements for the satellite control system according to the ground resolution, while the mapping satellites require the control system to provide accurate attitude information for each image.

As the exposure frequency of camera payload is generally within $10 \mathrm{~K}-100 \mathrm{~K}$, the measured attitude data (measurement accuracy: 1", measuring frequency: 10 $\mathrm{KHz}$ ) are needed in the theoretical calculation in order to realize 1:10,000 mapping at an orbital altitude of $500 \mathrm{Km}$. Therefore, this design scheme is of high degree of difficulty.

In the GF-7 satellite control system design, the task requirement characteristics are combined, and an eclectic and optimized design scheme is used to constrain the stability of the control system, in an effort to adapt to the current technological development status. Given this, a high-stability $\left(2 \times 10^{-40} / \mathrm{s}\right)$ system is designed to provide accurate attitude information within the testable frequency range; meanwhile, an $8 \mathrm{~Hz}$ high-precision (0.6") attitude measurement system is designed to provide absolute attitude accuracy data within a certain frequency range.

\subsection{Flutter analysis of the control system}

According to the decomposition of the control accuracy index, the indexes influencing the control system stability generally include the disturbance of rotatable satellite parts, measurement noise of actuator, design error of control law, system delay, control cycle, etc. The measurement accuracy mostly depends on that of the highprecision measurement sensor-star sensor.

The high-precision and high-resolution mapping satellite is usually equipped with the rotary solar array, and earthed data transmission antenna, and it is controlled by momentum wheels, which serve as the actuator.

In order to guarantee the high system stability, the disturbance caused by the rotary parts was tested, and the control law was designed through the analysis.

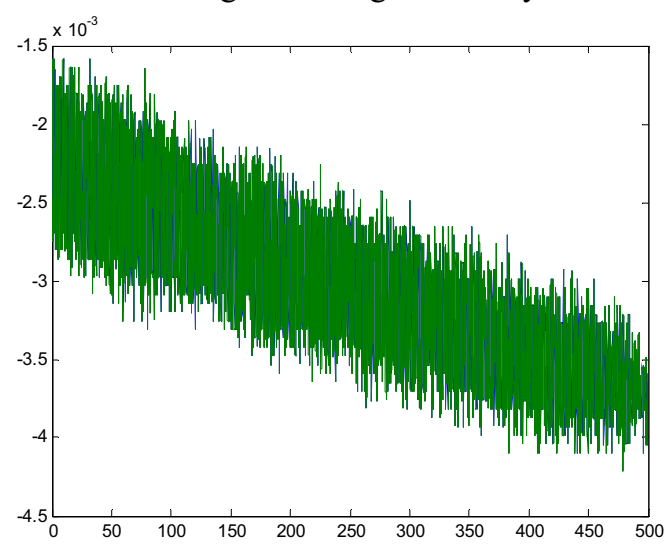

Fig.1 The influence of the rotary solar array on the system stability before optimizing

The influence analysis of the rotary solar array on the system stability was carried out. Obvious low-frequency signals could be observed in the system from the test data and spectral analysis results, as shown in Figure 1 and Figure 2.

In order to figure out the influence of high-frequency vibration, the vibration performance test of the momentum wheels was conducted, and the waterfall curve of each momentum wheel was given. The disturbance characteristics were mastered through the vibration analysis, the disturbance suppression algorithm for the solar array control, and the star control law were respectively designed.

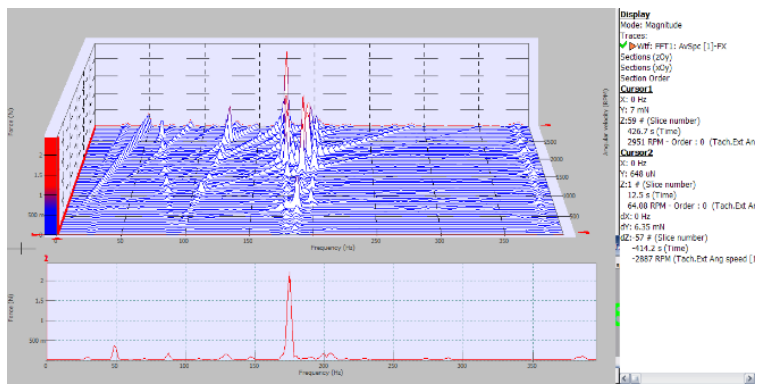

Fig 2. Spectral curve of the rotary solar array on the system stability 


\subsection{Control system configuration}

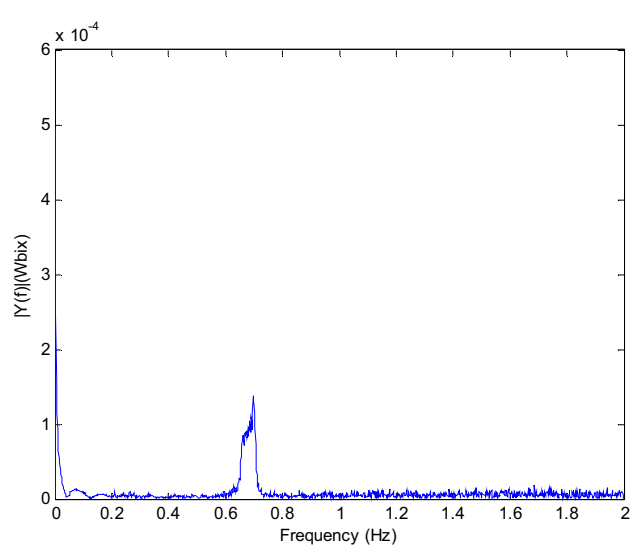

Fig.3 Waterfall curve of momentum wheels

Driven by the high-precision attitude measurement demand of the GF-7 satellite, an ultra-high-precision star sensor was newly developed in the control system, and combined with the three-floated gyro to realize the attitude measurement. Adapting to the demand for the reliability design, the IR ground sensor was designed in the measurement system to constitute a medium-precision attitude determination system together with the gyro, and thus realize the heterogeneous backup of the attitude determination system.

The momentum wheels were configured in the control loop design.

In order to overcome the flexibility aroused by the solar array driving during the imaging process, a highly steady solar array drive mechanism with the optimized control law was configured.

\section{Design Method}

\subsection{Attitude determination technique}

To reduce the low-frequency errors existing in the earlystage mapping satellite attitude, scholars in both China and foreign countries have investigated various algorithms in the last decade ${ }^{[5]-[7]}$, including the adaptive Kalman filtering method ${ }^{[8]}$, which identifies the short-periodic term coefficients in orbit through adaptive filtering, twostep two-way post-smoothing filtering algorithm [9], relative attitude measurement method [10], data postprocessing-based ground image compensation technique, etc. ${ }^{[11]}$ Its relative attitude measurement method relies upon the ground control point, while the two-step two-way post-smoothing filtering algorithm and ground image processing compensation method are more suitable for postprocessing.

In order to realize the attitude determination accuracy of 0.001 in the control system design for the GF-7 satellite, on the one hand, the ultra-high-precision star sensor with smaller short periodic errors was developed according to the index decomposition, which reduced the lowfrequency noise terms from the level of the standalone device; on the other hand, the attitude determination accuracy was further improved through various low- frequency error (LFE) suppression methods.

Stimulated by the index demand of the GF-7 satellite, the accuracy requirement of sub-arc second short periodic error was put forward for the first time, technological breakthroughs were made for the standalone device - star sensor, which ensured the structural thermal stability. The weight and power consumption of the star sensor were respectively constrained in order to improve the attitude measurement accuracy, which satisfied the demand for the integrated installation of satellite camera payloads, and reduced the variable quantity of the star sensor relative to the reference.

At the system level, the attitude parameter compensation method was applied to the GF-7 satellite to reduce the LFE of satellite attitude. According to the star sensor error classification ${ }^{[12]}$, this method was used to establish the Fourier multinomial series error model ${ }^{[13]}$.

The error terms of the star sensor at different frequency levels were respectively identified and compensated in the design. Directing at the LFE terms of the star sensor, the camera image data carrying control points in the GF-7 satellite were postprocessed, the LFEs in the satellite attitude measurement were identified through the extended Kalman filtering polynomial fitting parameter estimation method [14], and then compensated into the attitude measurement system. The compensation formula is as follow:

$$
\begin{aligned}
& \phi(t)=\sum_{k(t)}^{N}\left(a_{k x} \cos k \omega t+b_{k x} \sin k \omega t\right) \\
& \psi(t)=\sum_{k=1}^{k^{\underline{\underline{N}}}-1}\left(a_{k y} \cos k \omega t+b_{k y} \sin k \omega t\right)
\end{aligned}
$$

where $\phi, \hat{\theta}$ and $\psi$ denote the short periodic error angles in the directions of the roll, pitch and yaw, respectively; $\omega$ is the angular velocity of satellite operation; $t$ is time; $N$ is the total order of Fourier series in the star sensor LFE modeling; $a_{k i}$ and $b_{k i}(i=x, y, z)$ are to-be-determined Fourier series coefficients for the LFE.
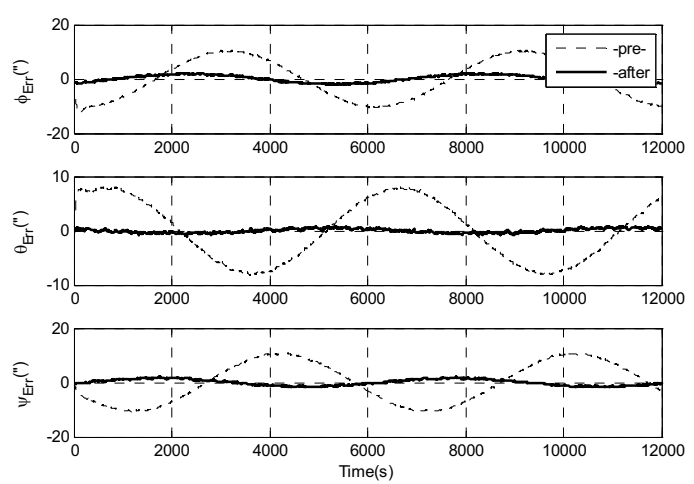

Fig.4 the comparision of attitude measurement accuracy between pre-compensation and after- compensation

\subsection{High stability control technology}

The distributed control technology was used for the GF-7 satellite control system to overcome the flexible vibration 
of solar array. The flexibility suppression design was carried out from two levels-standalone device and system - to improve the system stability. The functional block diagram of the distributed control is seen in Figure 5 .

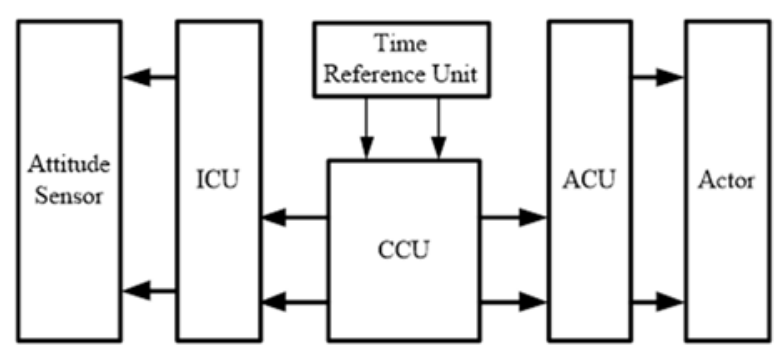

Fig.5 The diagram of distributed control

The optimized array drive mechanism was required to obtain a relative stability of $5 \%$, the flexible mode of the solar array was detected through the feedback elements installed at the output shaft, and then fed back to the controller of the drive mechanism, and the active control and suppression of the array disturbance were realized by designing a phase compensator. The transfer function $H_{1}(s)$ of the compensator is shown below:

$$
H_{1}(s)=\frac{\alpha T_{1} S+1}{T_{1} S+1}
$$

wherein $\alpha$ is graduation coefficient; $T_{1}$ is designed time constant.

\subsection{High-precision time synchronization system design}

A controller architecture with high time synchronization accuracy was designed in the GF-7 satellite control system. The time synchronizing signal connection is seen in Figure 8 . The control system receives the high-stability clock pulse per second (PPS) signal provided by the complete satellite via the CCU (Center Control Unit), sends it to the attitude measurement sensor (ICU : Information Control Unit) and actuator (ACU: Actuaor Control Unit), and meanwhile, calibrates the time reference inside the control system.

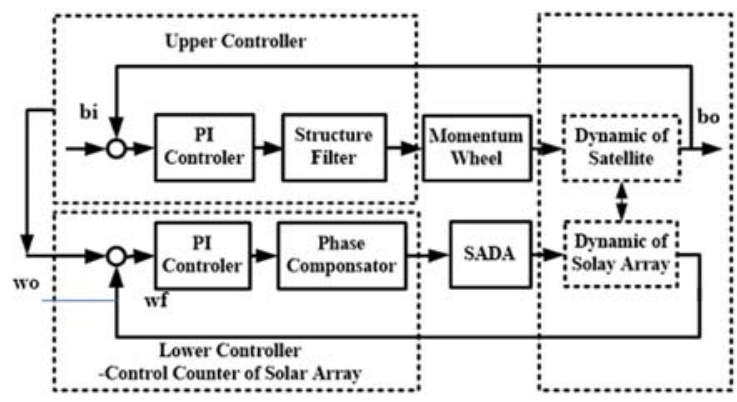

Fig.6 Logic diagram of time synchronization signal of GF-7 control system

In order to further improve the time synchronization precision, the uniform timing method is additionally used, besides the conventional clock reference unit timing ${ }^{[16]}$.

$$
\Delta t_{\text {timing }}=\Delta t_{\text {timing }} \times k+(1-k) \times \Delta_{\text {new }}
$$

The uniform timing formula is shown as below:

$$
\Delta_{\text {new }}=\left(T_{\text {new }}-T_{\text {old }}\right) / 1000000.0-1
$$

$\Delta t_{\text {timing }}$ is the time added by the control system in each control period. Wherein $T_{\text {new }}$ and $T_{\text {old }}$ are the satellite time counter values latched by the time reference unit PPS at the current time and the last time, respectively; The $k$ is the filter coefficient.

Before the PPS signal from the high-stability clock unit arrived the next time, the uniform adjustment and timing calculation of $<1 \mathrm{~s}$ satellite time were introduced into the system, namely uniform timing within the rest control period according to $\Delta t_{\text {timing }} / n$, where $n$ is the control period.

\subsection{Short-delay timing sequence design}

In the software timing sequence design, the timing sequence strategy with separate operation of attitude measurement and control law calculation was used to realize the optimized design of the system delay, and the controlled quantity was then rapidly output, being faster than the delay time before the optimization by tens of milliseconds. Therefore, the stability of the control system is further enhanced.

\section{In-Orbit Flight Validation}

The GF-7 satellite was launched on November 3, 2019, and the corresponding operating mode of the control system was validated according to the flight procedures. Through the analysis of in-orbit remote sensing data, the control system reached the indexes as required. The attitude stability reached $10^{-50} / \mathrm{s}$, and the inertial attitude measurement accuracy was higher than $0.001^{\circ}[17]$.

Table2 statistic of precision for star sensor on orbit

\begin{tabular}{lccc}
\hline error & $\begin{array}{c}\text { X_Axis” } \\
(3 \sigma)\end{array}$ & $\begin{array}{c}Y_{-} \text {Axis” } \\
(3 \sigma)\end{array}$ & $\begin{array}{c}Z_{-} \text {Axis" } \\
(3 \sigma)\end{array}$ \\
\hline Probe A & 0.4335 & 0.4518 & 8.7960 \\
Probe B & 0.5077 & 0.4951 & 10.1959 \\
Fusion & 0.2318 & 0.1889 & 0.5491 \\
Cross angle & & $-1.8 \sim 1.8(p-p)$ & \\
\hline
\end{tabular}




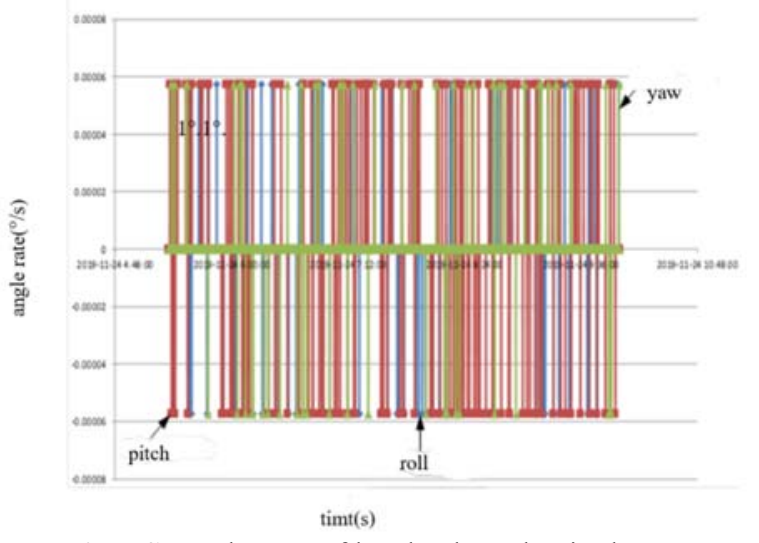

Fig.7 Control curve of local enlarged attitude rate

\section{Conclusion}

Following the in-orbit flight validation, the control system of the first high-resolution mapping satellite GF-7 meets the proposed high accuracy requirement. The distributed control technology used in the control system has effectively elevated the stability index. Furthermore, the newly developed star sensor has effectively mitigated the fluctuation of short periodic terms in the attitude measurement. It is not difficult to find from the in-orbit test data that the LFE terms presenting the orbital periodicity still exist in the star sensor, so the attitude determination accuracy should be further improved through the postprocessing technology according to the ground images in the follow-up research.

\section{Author}

Chenchao: Doctor of BUAA, and Researcher of BICE. Study of attitude control of satellite.

\section{Reference resource}

1. CAO H Y, LIU X G, LI S H et al. ZY-3 Satellite Remote Sensing Technology[J]. Spacecraft Recovery \& Remote Sensing,2012,33 (3): 7-16.

2. PAN T, GUAN H, HE W. GF-2 Satellite Remote Sensing Technology[J].Spacecraft Recovery \& Remote Sensing,2015 (4): 16-24.

3. ZHOU J M, WEI Y, CAO Y M et al. AOCS Design and On-orbit Verification of GF-3 Satellite[J]. Spacecraft Engineer.2017, 26 (6): 93-98.

4. GUO L H,YU X Q.The Summarize of Mapping Satellite Development of Foreign Country. [J], Mapping Technical Equipment.2013, 15 (3): 86-88

5. MO F,CAO H Y, LIU X G et al.Research on Space Mapping System with Large Scale[J]. Spacecraft Engineering,2017, 26 (1): 12-19.

6. Iwata $\mathrm{T}$, Hoshino H, Yoshizawa $\mathrm{T}$, et a1. Precision attitude determination for the advanced land observing satellite(ALOS): design, verification, and on-orbit calibration[C]. AIAA Guidance, Navigation and controlConference and Exhibit, South Carolina, August 2007.

7. WANG S Y,LIU X, CHEN C. Overview of Control System and on-Orbit Test for ZY-3 Satellite [J]. Aerospace Control and Application,2015, 41 (2): 15.

8. LIU Y W, CHEN Y Q. Star-sensor measurement model and its application to the spacecraft attitude determination system $[\mathrm{J}]$. Aerospace Control and Application.2003,24(2):162-167.

9. ZHAO L,XIE R D,LIU Y. Offline calibration method of low frequency error of star sensor and gyroscope drift[J]. Acta Aeronautica ET Astronautica Sinica, $201738(5): 42-50$

10. ZHANG C Q, WANG S Y, CHEN C. A HighPrecision Relative Attitude Determination Method for Satellite[J]. Aerospace Control and Application, 2014 (3): 19-24

11. Xie J F, Tang X M, Mo F et al, Domestic Stellar Image Post-processing Project Design and System Implementation for ZY-3 Satellite[J].Geomatics and Information Science of Wuhan University, 2017, 42(4):434-439

12. LU X, WU Y P. Low Frequency Error Analysis of Star Sensor[J]. Aerospace Control and Application,2014 40(2):1-7.

13. XIONG K, ZONG H,TANG L.Calibration of Star Sensor's Low Frequency Error Based on Landmark Information[J]. Aerospace Control and Application, 2012, 38(3):11-15

14. WANG S Y,LIU X, CHEN C.The Study of Postprocessing-based ground image compensation technique of ZY-3 Satellite, [C].Papes of 19th CHINA TeleSensor Conforence: 361-366

15. ZONG $\mathrm{H}$, ZHANG $\mathrm{H} \mathrm{H}$, XU $\mathrm{F} X$. Angular Momentum Feedback Control for Disturbed Flexible Satellites[J]. AEROSPACE CONTROL, 2009,27(6): 42-51

16. WANG Y, ZHAN Y, CHEN C. A High-Precision Timing System of AOCS Based on Synchronous Pulses Latching and Processing[J].Aerospace Control and Application,2014 40(6): 26-30

17. CHEN C,DING J Z,WANG S Y,LIU J.High precision control technology and on orbit verification of GF-7 satellite[J].2020,VOL 40.Issue(5):40:34-41 